Free Incision" in one of the journals, and I may add that subsequent experience has but confirmed me in the impression I had then formed. A free cut into a suppurating or disorganised joint, whether associated or not with bone disease, is rarely followed by any other than a good result. When the suppurating process is due to synovial disease, a recovery without further surgical interference may be looked for. When due to local necrosis the incision helps nature towards the recovery of the cases by expediting the process of exfoliation, and the subsequent removal of the bone by either natural processes or some surgical proceeding. And in still more severe cases the incision gives relief, and in no way tends towards inducing any increase in the mischief.

(To be continued.)

\section{ON JABORANDI.}

\section{BY SYDNEY RINGER, M.D.; AND \\ ALFRED P. GOULD, M.B., B.S., UNIVERSITY MEDICAL gCHOTAR.}

THIs active and interesting drug, long in use in South America, was lately brought from Pernambuco to Paris by Dr. Continho. Dr. Gubler and M. Rabuteau confirm Dr. Continho's statement concerning its powerful diaphoretic and sialagogue properties. The dose for an adult is sixty to ninety grains, infused in boiling water. Dr. Continho recommended that the patient should drink the infusion with the suspended leaves, and then go to bed and cover himself with warm clothing. In about ten minutes the face, ears, and neck become deeply flushed; then soon drops of perspiration break out over the body, and at the same time the mouth waters; in a short space the perspiration rapidly increases, the sweat running down the body and soaking the clothes, whilst the salivation becomes profuse, running from the mouth sometimes in an almost continuous stream. In three of our cases the saliva amounted to an imperial pint, $22 \mathrm{oz}$., and $27 \mathrm{oz}$. respectively. The sweating and salivation continue from two to four or five hours, and simultaneously decline. Dr. Gubler has shown that the drug increases the bronchial secretion, and he further asserts that it sometimes produces diarrhoea. In a communication concerning the action of this medicine on some lads between the ages of eight and twelve lately published in the Practitioner, we confirmed these statements, with the exception of that relating to diarrhoea, and drew attention to the influence of this drug on the temperature, pulse, brain, \&c. During the sweating we found that the temperature fell about one degree, the reduction persisting for the rest of the day; and that the lowest temperature was reached about an hour and a half after the administration of the medicine. In one case the fall began immediately, in another case in forty minutes, and in another instance in eighty minutes. In each experiment the pulse became considerably quicker, the increase varying from forty to fifty beats, the beats being quickest in from twentyfive to eighty minutes after the dose. The quickened pulse continued more than four hours. In one lad with intermittent pulse the medicine entirely removed this irregularity. The drug of ten produced nausea or sickness, which occurred two or three hours after taking it; it also occasioned considerable drowsiness, the lads falling asleep during the observations. The flushing of the face was noticed only when sweating occurred; and in a short time the flushing was succeeded by marked pallor, accompanied by some prostration. We tried to show that probably the fall of temperature was due to the perspiration and to increased radiation of heat from an increased quantity of blood flowing to the skin.

Recently M. Albert Robin has published the results of his investigations. He points out that, in addition to the sweating and salivation, the lachrymal and nasal secretions are increased; and asserts that before and during the sweating the temperature rises more than from $1^{\circ}$ to $2^{\circ} \mathrm{F}$., and then becomes normal; and that after the sweating the tem- perature falls more than from $1^{\circ}$ to $2^{\circ} \mathrm{F}$., and the depression lasts for a day or two. Sphygmographic tracings taken at different stages of the experiment proved that during sweating jaborandi produces very notable diminution of vascular tension. After the cessation of the sweating the pulse was much lowered.

We have recently made thirty-seven careful observations: twenty on adults, whose ages varied from twenty to fortyfive; and seventeen on children, between three and ten years of age. To the adults we gave sixty grains; to the children thirty, and sometimes sixty. These investigations have led to the detection of several new and interesting effects of jaborandi. They lead us to conclude that some of M. Robin's statements are inaccurate. They show that children are very little affected by this drug. We administered generally thirty, sometimes sixty grains, giving both the solution and the dregs; and yet, strange to say, this dose often failed to produce any effect. In two cases only was the sweating abundant; in eight it was slight - in most, very slight, making the skin only a little moist; and in seven cases it excited no perspiration. Salivation was generally absent, and never more than to make the mouth rather moister than usual. There was very little flushing of the face, and this occurred only when perspiration was induced. The pulse was but slightly increased in frequency, from ten to sixteen beats, and this occurred only when the remedy acted on the skin. When the pulse was quick (and several of these children were convalescent and weak), we noticed that the pulse was still less influenced, and sometimes even fell. In our earlier observations on older children, where the effects were well marked, the pulse was much more quickened. When the skin was rendered moist, the temperature generally fell, the fall varying from $0.6^{\circ}$ to $2^{\circ} \mathrm{F}$. In eight of these cases it is noted that the child vomited, usually two hours after the administration of the medicine, but sometimes sooner Occasionally the vomiting was repeated three times. When the vomiting was Jong delayed none of the dregs of the medicine were rejected. Many of the children were sleepy during the stage of depression. In no instance were the bowels affected, nor did the medicine excite any cough. To sum up : in many cases the children were not at all affected in their rest; the symptoms were very slight, most strikingly so when compared with the marked effects of jaborandi on adults.

In the twenty observations made on eighteen adults, the perspiration in every case but two was most profuse, and in these exceptional instances it was free. It began in about ten minutes, and in one instance only was delayed for an hour. Salivation was absent in two cases only; in three cases it was slight; in the rest profuse, amounting in one experiment to $27 \mathrm{oz}$. In one case only the pulse was unaffected, though there was marked perspiration. In the rest the pulse was quickened from 12 to 40 beats, the average being 20 beats per minute; the average increased frequency lasting two hours and a half.

The temperature was taken in nine cases, three in the axilla, the rest under the tongue. In every instance the temperature fell during the sweating, the fall varying from $0.4^{\circ}$ to $1.4^{\circ}$, the average being $0.9^{\circ}$, and lasting from one hour and a quarter to four hours and a half, and then becoming normal; that is, the temperature reached the point observed before the medicine was given. This was administered between 9 and 10 A.M. The average duration of the fall was three hours and a quarter. In one instance only did the temperature rise, and then only $0.4^{\circ}$, the rise lasting a very short time. On this point our observations are opposed to those of M. Robin, who states that the temperature rises at first, and then remains depressed two or more days. This statement being quite at variance with our observations (for we have said that in a few hours the temperature recovered itself), we noted the temperature in three men every three hours for three days before the administration of the drug, every hour during the action of the medicine, and every two hours for two days after the medicine. We found that in a few hours the temperature returned to the point noted before the drug was given, and on the two following days was the same as on the two days previous to the experiment. In one case only the flush of the face and neck was absent, and in every case save two the flush was followed by pallor and more or less depression or even prostration. In half the cases we noticed slight cough due 
to increased bronchial secretion, but this was always slight. In no instance did the medicine relax the bowels, so that its action on the intestinal mucous membrane must be slight. In one-half the cases the patients became decidedly sleepy, this occurring during the pallor, one, two, or three hours after the administration of the drug. A sensation of sickness, and sometimes decided nausea, occurred in two-thirds of the cases. In several cases the sight was decidedly affected. Mr. Martindale first drew our attention to this fact. He took, experimentally, a free dose of the medicine, and we saw him an hour and a half afterwards, when he said everything at a little distance looked hazy. On testing his sight we found that he could read moderate-sized type at about a foot distance, but at two feet the type became indistinct. This led us to investigate the effect of jaborandi in this respect in other persons. In three other cases, after the internal administration, the sight became decidedly affected; but the size of the pupil was unaltered. Mr. Martindale prepared for us an extract dissolved in glycerine, which we introduced into one eye of thirty-one persons. In nineteen cases the pupil became decidedly contracted, the amount of contraction varying, in some being slight, in others reduced by one-third, in two instances to one-half. We noticed that the pupil acted sluggishly in eleven cases. In a few hours the contracted pupil regained its natural size; but in one case the contraction was noticed twenty hours after the application. M. Robin finds that the tears and the nasal secretion are increased; but in four only of our cases were the lachrymal and nasal secretions increased sufficiently to attract our attention or that of the person experimented on. In eleven cases complaint was made of pain over the pubes, and in some instances this was severe, and was generally accompanied by a strong desire to pass water, which a fiurded relief. A good deal of trembling, especially of the upper extremities, followed in two instances after the administration. This was the case with Mr. Martindale, which did not appear to be due to nervousness. One man complained of much throbbing of the head the day following the experiment. Many patients complained of feeling alternately hot and cold, or chilly.

It will be seen that our later observations prove a marked antagonism between jaborandi and belladonna. Belladonna checks the secretion from the skin, the salivary glands, the mucous membrane of the nose, bronchial tubes, stomach, and intestines, and dilates the pupil and contract the arterioles; jaborandi, on the other hand, increases enormously the perspiration and saliva, and, in a much less degree, the secretion from the mucous membrane of the nose, the bronchial tubes, and the stomach and intestines. Robin asserts, moreover, that it lowers arterial tension, probably by paralysing the vaso-motor nerves, and thus causing dilatation of the arterioles. Belladonna excites delirium, whilst jaborandi of ten produces sleep. This antagonism was well displayed in four instances. On three occasions, to three different men, we administered sixty grains of jaborandi, and when the perspiration and salivation were profuse we injected subcutaneously $\frac{1}{10 \sigma}$ of a grain of atropia. In five minutes the perspiration and salivation were considerably diminished, and in from ten to twelve minutes the skin was preternaturally dry and the salivation was checked, but in one case continued a little after the cessation of the perspiration. It curiously happened that a lad was admitted into University College Hospital poisoned by atropia. He had taken one grain of the alkaloid five hours before treatment. His tongue and mouth were quite dry. A quarter of an hour after taking thirty grains of jaborandi his mouth became much less dry, and in an hour he was sweating freely and his mouth was naturally moist.

Belladonna, it is well known, arrests the secretion of milk, and we were anxious to ascertain whether jaborandi was antagonistic to belladonna in respect to its influence on this secretion. We administered thirty grains of jaborandi to a woman thirty-eight years old, confined of her ninth child four months previously. During suckling she had very little milk, and the quantity had become much less of late. We gave her the medicine at 10 A.M. She had suckled her child seven hours before. In ten minutes the drug produced its usual symptoms; in half an hour her breasts, which previously were flaccid, became tumid and distended, and on pressure yielded considerably more milk. In forty minutes the increase was still more marked, jetting forth in four or five streams. In eighty minutes the milk was less abundant, though considerably more so than before the experiment. The woman herself noticed the decided increase of her milk. To another woman, aged twenty-five, whose child is thirteen months old, we gave two doses of thirty grains, as the first had no effect. She emptied her left breast every ten minutes by pressure, and each of the three first emptyings yielded 40 minims. As soon as perspiration and salivation became free the quantity rose to 80 minims. The next time yielded 100 minims, the following 155 minims, the next time 80 minims. The salivation and perspiration at this time ceased. The next observation yielded 125 minims, the next 87 minims, the next 70 minims, and the last 40 minims. This woman, whose case is not included in those given before, complained of severe pain over the pubes, with desire to urinate, the pain immediately ceasing after micturition.

In some of their effects, however, jaborandi and belladonna agree. Thus, both flush the face and quicken the pulse: and both affect children much less than adults. Belladonna quickens the pulse by its paralysing influence on the pneumogastric, and possibly by stimulating the sympathetic nerves. We have no evidence that jaborandi paralyses the pneumogastric, or stimulates the sympathetics supplying the heart, but $M$. Robin has shown that it lessens arterial tension, probably by dilating the arterioles; and it is $w$ ell known that dilating the arterioles, and thereby allowing the blood to pass more quickly from the arteries to the veins, quickens the heart-beats. Hence it is very probable that the quickening of the pulse by jaborandi is due to its influence on the arterioles; and thus, instead of affording an instance of similarity of action between these two drugs, affords another example of their antagonism, for belladonna contracts the arterioles. While referring to the relations of these two drugs, we may point out that both sometimes affect the bladder. Jaborandi, as we have seen, causes pain over the pubes, with desire to pass water, the pain subsiding when the bladder is emptied. These facts render it probable that jaborandi causes contraction of the bladder, for it does not augment the quantity of the secretion.

Jaborandi probably paralyses the sympathetic nerve, the effect being shown by the deep flushing of the face. Mr. Robin ascribes its effects to its influence on the sympathetic. But there are various reasons to doubt this conclusion. It is improbable that mere increase of blood could cause so great an increase of sweat and saliva. Belladonna flushes the face, but completely arrests the sweat. Again, it has been experimentally shown that belladonna will check the secretion from the submaxillary gland, although by irritation of the chorda tympani nerves the bloodvessels supplying the gland are dilated and full. It is true the flushing from jaborandi immediately precedes the outbreak of perspiration, but soon the flush declines, and is succeeded by marked pallor, yet the perspiration continues for hours, being often even more abundant than during the flush. To test whether the effects of jaborandi wore off by repetition, we administered sixty grains to a man on three consecutive days, and the symptoms were as marked on the third as on the first day. On each day, in a few hours, the temperature recovered from the depression, rising to the height observed before the medicine was given. We should have continued this investigation some days longer, but the man appealingly protested that we were giving him more than his fair share of experimentation. In two other cases we gave sixty grains on two consecutive days, and the effects were as marked on the second as on the first. In one of these cases the temperature was observed, and on both days the temperature in a few hours recovered from its depression. These cases strikingly show the erroneousness of M. Robin's assertion that jaborandi causes a depression on the day following. On the other hand, we found the effects soon ceased in children, and sometimes the second dose failed to excite perspiration.

It may be suggested that the perspiration was due to the nausea and vomiting excited by the drug. Those who witnessed the effects of this medicine will certainly not ascribe them to the depression from nausea; but we may add that the perspiration was too profuse to be due to mere depression and nausea. Moreover the perspiration occurred often some hours before the nausea or depression set in. Again, in children, though the drug in some cases caused much and repeated vomiting, there was no perspiration; moreover, in many cases, especially among adults, the full phy- 
siological effects of the drug were obtained without either nausea or depression.

We have tried in several cases a tincture from the leaves, and in one case an extract made from the tincture, and found these preparations equally effectual as an infusion with the dregs. We find also that a strained infusion is equally efficacious as one with the dregs, from which we conclude that the active principle is not the oil, so abundant in these leaves. In one case we administered a strained infusion of the root without any effect, and in three cases an infusion made from the bark of the stem, giving also the dregs. This last preparation is also active, though it appeared to us to excite more vomiting and to cause more depression than the preparations from the leaves.

The following account of the effects of jaborandi on the eye is written by Mr. JoHN TwerdY :-

"From the account given in the Pharmaceutical Journal of the 16th inst., by Mr. Martindale, of his personal experience of the physiological effects of jaborandi, it appeared that in addition to its diaphoretic and sialagogic actions, this drug was capatele of exerting a decided influence on the accommodative apparatus of the eye. About half an hour after taking fifty grains of the powdered leaf in an infusion, Mr. Martindale found that his vision for distance was greatly impaired, although he could see near objects distinctly. It was stated that the pupils were slightly dilated during this time, but Dr. Ringer, who was called in, informs me that this statement was not correct.

"In order to ascertain, if possible, what effect jaborandi really had upon the eye; some of the extract was placed within the conjunctivæ of several patients. Of its effects on the pupil full particulars have been given above. My attention was directed more particularly to the state of vision and the accommodation, and to the opththalmoscopic appearances of the fundus oculi. I must, however, confess that as far as the six or seven patients that I examined were concerned I failed to find any constant or definite changes in the appearance of the eye beyond contraction of the pupil. One thing, however, was noticed - namely, that whereas the details of the fundus of the unaffected eye could be seen clearly without a lens behind the mirror of the ophthalmoscope, a weak concave lens was necessary to see clearly the details in the eye experimented on, thus showing some tension of accommodation.

"Failing to elicit any definite or satisfactory information respecting the condition of the accommodation of the eye, in these patients, I determined to apply some of the extract to my left eye, and to note its effects. Accordingly, at 1.30 P.M., I carefully tested the state of vision of my left eye, and found that I could read easily and clearly No. ${ }^{1}$. Snellen's type from $4 \mathrm{in}$. (my nearest point) to $22 \mathrm{in}$. (my farthest point), and that $V=\frac{10}{10} ;$ i. e., at a distance of $10 \mathrm{ft}$. I could read No. 10 of Snellen's type. (It may be well perhaps to state for the benefit of those not acquainted with Snellen's test-type, that whenever I shall express the state of vision or $V$ by a fraction, the numerator will represent the distance at which I stood from the type, and that the denominator will represent the size of the type seen at that distance, or, more correctly, the distance at which that type makes an angle of five minutes at the optical centre of the eye.) I next made my left eye myopic by placing before it a convex lens of ten inches focal length, and found that $I$ could see clearly and distinctly the fine vertical line of the dial I employ for estimating the degree of astigmatism, at a distance of nine inches. At $1.45 \mathrm{I}$ placed within the conjunctiva a drop of the extract of jaborandi, which produced smart pain for about five minutes. After the smarting and the lachrymation had ceased, I tested the state of vision of the eye, and found that at 2.0 the nearest point of distinct vision for $1 \frac{1}{2}$ Snellen had been approximated to $3 \frac{1}{4}$ in., and the farthest point to $20 \mathrm{in}$., while the fine vertical line could not be clearly seen, with the convex 10 in., at a oreater distance than 81 in. At 2.15 the nearest point for $1 \frac{1}{13}$ Snellen was at 3 in., and the farthest at 14 in., and the greatest distance for the vertical line, with convex $10 \mathrm{in.}$, was $8 \mathrm{in.}$ I could, at a distance of $10 \mathrm{ft}$., make out some of the letters of $\mathbf{1 2}$ Snellen, but not even the letters of 30 Snellen were quite clearly defined. Just below the lower margins of each of the letters of 30 Snellen I could wee, as it were, a fine shadow of half of each letter. There was at this time no perceptible contraction of the pupil. At 2.25 the nearest point for $1 \frac{1}{3}$ Snellen was at 3 in., and the farthest point at 12 in. The left pupil was now noticed to be contracted to about half the size of the right. At 2.30 I could scarcely make out any of the letters of 15 Snellen at $10 \mathrm{ft}$., and even 20 Snellen were not clear. I now observed that even those letters that I could make out appeared much smaller when viewed with the left eye than with the right, and that with the left eye they seemed to be at a greater distance from me than with the right. At 2.35 the nearest point for $1 \frac{1}{2}$ Snellen remained at 3 in., but the farthest point had receded to $15 \frac{1}{2}$ in. The inequality of the pupil was in statu quo. At 2.40 I noticed that all objects, but especially distant ones, appeared much bolder, brighter, and better defined with my right eye than with the left, although under ordinary circumstances the vision of the left eye is more acute than that of the right. There appeared to be, in addition to the short-sightedness of the left eye, an actual impairment in the sensibility of the retina, producing imperfect amblyopia. At 2.50 the nearest point for $1 \frac{1}{2}$ Snellen was at $3 \frac{1}{4}$ in., and the farthest at 16 in. The fine vertical line could now be clearly seen with the convex 10 at $8 \frac{1}{8}$ in. At 2.55, $\nabla=\frac{1}{1} \frac{0}{2}$, and I could now decipher some of the letters of No. 10, all the letters being clearer and better defined. The inequality of the pupils, however, remained the same. At 3, I could read even No. 8 Snellen at $10 \mathrm{ft}$., and there was but little appreciable difference in the apparent size or distance of the letters when viewed with the right and left eyes alternately. The left pupil had relaxed a little, but was still smaller than the right. At 3.10 P.M. the nearest point for $1 \frac{1}{2}$ Snellen was $3_{4}^{3}$ in., and the farthest point at 18 in. The vertical line could now be seen at 9 in. with convex 10 in. At 3.30 the vision of the left eye had resumed its normal state, but it was not till after four o'clock that the left pupil had thoroughly relaxed.

"From these facts we may conclude that jaborandi locally applied to the eye causes, (1) contraction of the pupil; (2) tension of the accommodative apparatus of the eye, with approximation of the nearest and furthest points of distinct vision; (3) amblyopic impairment of vision from diminished sensibility of the retina. These effects, however, do not last long. In my own case the approximation of the near and far points of distinct vision had declared itself in a quarter of an hour, and reached its maximum in about forty minutes. It then gradually subsided, and had entirely passed off and the eye resumed its normal state in about an hour and a half."

\section{AUTOPSY OF THE TRING CENTENARIAN} WHO HAD ATTAINED THE AGE OF ONE HUNDRED AND ELEVEN YEARS.

By Sir G. DUNCAN GIBB, BaRT., M.D., LL.D., PHYSICIAN TO THE WRSTMIXSTER HOSPTMAL.

AN account of the autopsy of the old dame Mrs. Elizabeth Leatherlund, known as the Tring or Hertfordshire centenarian, just deceased, who was within three months of attaining the great age of one hundred and twelve years, will be most fittingly recorded in the pages of 'THE Lancer. of the correctness of her age, as I have already had occasion to state in a communication brought before the British Association at Belfast in August of last year, there is no doubt, and very shortly a paper of mine, embodying all the proofs of this, will be brought before one of our scientific institutions. But it may be stated here, that she mentioned she was born at Chinnor, in Oxfordshire; she gave the christian name of her father correctly as well as her own; her maiden name she described as Herne, although spelt Horam in the Parish Register of Baptiems at Chinnor* for 1763 , in which occurs this:- "Elizabeth, daughter of Thomas Horam, Travailer, April 24." No subsequent entry occurs in this Register for thirty-seven years, giving her own or father's name, which disposes of an objection to her age that wus brought for-

* Through an error last year, Dover was stated in place of Chinnor. 